\title{
Can We Boost Preschoolers' Inhibitory Performance Just by Changing the Way They Respond?
}

\author{
Daniel J. Carroll (iD and Emma Blakey \\ University of Sheffield
}

\author{
Andrew Simpson \\ University of Essex
}

\begin{abstract}
Changing the way children make their response appears to sometimes, but not always, boost their inhibitory control-though interpreting existing findings is hampered by inconsistent methods and results. This study investigated the effects of delaying, and changing, the means of responding. Ninety-six preschoolers $\left(M_{\text {age }}\right.$ 46 months) completed tasks assessing inhibitory control, counterfactual reasoning, strategic reasoning, and false belief understanding. Children responded either immediately or after a delay, and either by pointing with their finger, or with a hand-held arrow. Delaying boosted performance on all tasks except false belief understanding; arrow-pointing only improved strategic reasoning. It is suggested that delay helps children work out the correct response; it is unlikely to help on tasks where this requirement is absent.
\end{abstract}

Inhibitory control is the ability to overcome prepotent but task-inappropriate responses. Overcoming these responses is central to the ability to act freely on the world, and inhibitory control is vital to many aspects of development, including mental-state reasoning (Carlson \& Moses, 2001), counterfactual thinking (Beck, Riggs, \& Gorniak, 2009), academic performance (Gilmore et al., 2013), and resisting temptation (Murray \& Kochanska, 2002). The ability to overcome these responses improves rapidly during the preschool period (Petersen, Hoyniak, McQuillan, Bates, \& Staples, 2016), partly through improvements in inhibitory control itself (Gerstadt, Hong, \& Diamond, 1994), but also via other routes, such as better regulation of the speed of responding, and cognitive distancing (Lee \& Atance, 2016). Understanding the nature of these alternative routes is essential, both for understanding how inhibitory control develops and as a basis for developing interventions to help children who have difficulty with self-regulation.

Because inhibitory control is required in so many domains, there is widespread interest in finding ways in which young children-whose inhibitory control is poor-can be helped to overcome incorrect prepotent responses. Indeed, an intriguing (though fragmentary) literature offers promising findings suggesting that it is possible to boost the ability to overcome an incorrect prepotent response

Correspondence concerning this article should be addressed to Daniel J. Carroll, Department of Psychology, University of Sheffield, Sheffield S1 2LT, United Kingdom. Electronic mail may be sent to d.carroll@sheffield.ac.uk. using simple interventions. There are two distinct manipulations that appear to help children. Both focus on the way in which children make their response.

The first such manipulation is to delay children's responding. Because the speed at which preschoolers respond is highly variable (Eckert \& Eichorn, 1977), delay manipulations have tended simply to add an extra step to the standard task responsefor example, by singing a song (e.g., Ling, Wong, \& Diamond, 2016), or waiting for an unrelated event to finish (e.g., Beck, Carroll, Brunsdon, \& Gryg, 2011). The rationale for this approach is that delaying responding reduces a task's inhibitory demands by providing additional time-either for the correct response to be worked out (the Active Computation account: Diamond, Kirkham, \& Amso, 2002), or for the activation of the incorrect response to fade (the Passive Dissipation account: Simpson \& Riggs, 2007).

The second manipulation changes the way children make their response, typically replacing a finger-pointing gesture with an unfamiliar actionfor example, pointing with an arrow (Beck et al., 2011), or placing a hoop over an object (e.g., Simpson \& Riggs, 2007). The broad explanation behind

\section{(c) 2021 The Authors}

Child Development published by Wiley Periodicals LLC on behalf of Society for Research in Child Development

This is an open access article under the terms of the Creative Commons Attribution License, which permits use, distribution and reproduction in any medium, provided the original work is properly cited. All rights reserved. 0009-3920/2021/9206-0022

DOI: $10.1111 /$ cdev.13617 
this approach is that unfamiliar means of responding create "cognitive distance," a putative shift in perspective that allows children to view their own actions as a subject for reflection (Hala \& Russell, 2001), making unreflective, impulsive errors less likely. We refer to these response-based improvements as Response Delay effects and Response Mode effects, respectively.

There is evidence to suggest that both kinds of response manipulation can help children to overcome incorrect prepotent responses. But surprisingly, given the importance of the topic, the available evidence is scant, and results are inconsistent. Because methodologies vary greatly across studies, it remains unclear whether inconsistencies are due to the phenomena themselves being unreliable, or due simply to incidental task differences.

For example, introducing a delay improves children's performance on some inhibitory control measures, but not others. On the Day-Night task, making children wait until the experimenter has sung a song helps children inhibit the prepotent response (Ling et al., 2016). But on Go/No-go tasks, delaying responding by placing a screen between the child and the array does not boost performance (Barker \& Munakata, 2015). Both tasks tap inhibitory control, but adding delay leads to different outcomes. Inhibitory control also plays an incidental but crucial role in many reasoning tasks (e.g., Beck et al., 2009; Carlson \& Moses, 2001; Sabbagh, Moses, \& Shiverick, 2006). Delaying helps children on some of these reasoning tasks, but not others. Children's counterfactual reasoning (e.g., answering the question, "If the wind hadn't blown, would the picture be on the table, or in the tree?") improved when responding was delayed (Beck et al., 2011). However, delay did not improve performance on strategic reasoning tasks: when children had to point to an empty box in order to win a box containing a reward, they did just as badly after a delay as when they responded immediately (Hala \& Russell, 2001). Both tasks require children to suppress a prepotent response; if delays help children to overcome such a response, then we should expect consistent improvement. Thus, results across tasks are inconsistent and hard to interpret, leaving open the question of whether the Response Delay boost is a generalizable phenomenon, or a taskspecific artifact.

Similarly, inconsistent methodologies and findings are seen with Response Mode manipulations. Making children respond by pointing with an arrow (rather than with their finger) helps overcome prepotent responses on measures of counterfactual reasoning (Beck et al., 2011), but not mental-state reasoning (Carroll, Riggs, Apperly, Graham, \& Geoghegan, 2012). This divergence is surprising: in both counterfactual tasks and theory of mind tasks, children must think about an alternative world, suppressing any tendency to respond based on reality. In contrast, many kinds of unfamiliar response modes help children on strategic reasoning tasks. Simply pretending to point with an arrow-while really pointing with their fingerboosted performance to near-ceiling levels (Carroll, FitzGibbon, \& Critchley, 2014). So unfamiliar response modes can provide a boost for preschoolers' inhibitory control-but seemingly only in certain contexts.

If delaying, or changing, the way children respond genuinely boosts inhibitory performance, it could offer a way to support children in situations where weak inhibitory control lets them down. However, it is still unclear whether either manipulation genuinely improves children's performance, or whether the disparate literature merely reflects an ad hoc collection of task-specific findings. Previous methodologies have varied greatly between studies. Many response manipulations have been used, including pointing with a rotating arrow (Carlson, Moses, \& Hix, 1998), or handheld arrow (Carroll et al., 2014); placing markers (Hala \& Russell, 2001); putting hoops over objects (Simpson \& Riggs, 2007); or pressing buttons (Russell, Hala, \& Hill, 2003). The range of delay manipulations is similarly wide, including singing the task rules (Diamond et al., 2002); singing a nonsense ditty (Ling et al., 2016); sitting in silence (Hala \& Russell, 2001); watching dolls go down slides (Beck et al., 2011); asking children to stop and think (Wimmer \& Perner, 1983); waiting for a screen to be removed (Barker \& Munakata, 2015); or giving oneself a tight hug (Kusche \& Greenberg, 1994). A systematic approach to testing these phenomena is needed, to identify whether these apparent effects are reliable.

To achieve that goal, this study tested the effects of response delay and response mode on a single sample of preschoolers, across four different cognitive domains, each measure of which involved overcoming an incorrect prepotent response. The study used a $2 \times 2$ design to compare the effect of manipulating Response Delay and Response Mode on a classic measure of inhibitory control, and on measures of strategic reasoning, counterfactual reasoning, and false belief understanding (where inhibitory control is required incidentally to suppress a task-inappropriate response). These tasks all require children to respond by selecting one of two 
locations-either immediately or after a delay; and either pointing with their finger or with an arrow (see Table S1 for a summary of other task demands). Children completed all four tasks, responding in one of four ways: Finger-Delay, Finger-No Delay, Arrow-Delay, and Arrow-No Delay. In the two No Delay conditions, children responded at their own pace; in the two Delay conditions, preschoolers responded by tapping the table three times, and then making their response. This novel manipulation was chosen as it avoids previous confounds by not providing rule reminders (see Barker \& Munakata, 2015), and because pilot testing showed that children found this way of responding easy. In the two Finger conditions, children pointed with their finger; in the two Arrow conditions, children responded using a hand-held arrow (a fast, nonstandard response: see Carroll et al., 2014).

\section{Method \\ Participants}

An a priori power analysis using G*Power (Faul, Erdfelder, Lang, \& Buchner, 2007) indicated that 88 participants were required to have $80 \%$ power for detecting a medium-sized effect $(0.25)$ for the two main effects (Response Mode and Delay), with an alpha value of .05 . Ninety-six typically developing, English-speaking children participated $\left(M_{\text {age }}=\right.$ 46 months, range $=39-54$ months; 39 males). Of these, 15 children failed to complete a full set of trials for the Black-White task. Children were recruited from preschools in medium-sized towns in two lower-middle socioeconomic areas of the United Kingdom. The group was predominantly White $(90 \%)$ and of mixed social class.

\section{Design}

A mixed $2 \times 2 \times 4$ design was used, with Response Mode (Finger vs. Arrow) and Response Delay (Delay vs. No Delay) as between-child factors, and task (Black-White task, Windows task, Counterfactual Reasoning task, False Belief task) as a within-child factor. There were four conditions: Finger-Delay, Finger-No Delay, Arrow-Delay, and Arrow-No Delay (all conditions: $N=24$ ). Children were randomly allocated to a condition, and used the same means of responding on all four tasks (see Appendix S1, instructions). A one-way analysis of variance (ANOVA) indicated that the conditions did not differ in age, $F(3,92)=11.20, p=.616$
$\left(M_{\text {age }}\right.$ in months: Finger-Delay 46.2; Finger-No Delay 45.2; Arrow-Delay 46.7; Arrow-No Delay, 45.7). Conditions were identical, apart from the way in which children made their response.

\section{Materials}

For the Arrow conditions, a small arrow mounted on a stick was used. For the Black-White task, black and white laminated cards were used. For the Windows task, four plastic boxes were used (two opaque, two transparent); colorful stickers were used as rewards. For the Counterfactual Reasoning task, four series of descriptive pictures were used, showing the key events for each story, as well as two possible answers. For the False Belief task, four series of descriptive pictures were used, showing the key events for each story, as well as two possible answers.

\section{Procedure}

Tasks were administered over two sessions in a counterbalanced order, constrained so that (a) the Windows and Black-White tasks were in separate sessions, and (b) the Counterfactual Reasoning and False Belief tasks were in separate sessions (see Table S2). Sessions lasted approximately $15 \mathrm{~min}$, and the two sessions were conducted on different days. Children used the same mode of responding for all four tasks, determined by their condition.

\section{Black-White Task}

This task was adapted from Simpson and Riggs (2009). Children were shown a black card and a white card, and were told to point to the black card when the experimenter said "white," and to point to the white card when the experimenter said "black." There were four practice trials with feedback, followed by 12 test trials without feedback, presented in a fixed pseudorandom order (WBBWBWWBBWBW).

\section{Windows Task}

This task was adapted from Carroll et al. (2012). For the training phase, children were shown two opaque boxes, and watched the experimenter hide a sticker in one of the boxes. Children were told that they could choose which box the experimenter looked in, and that if the experimenter found the sticker, she would keep it; the child could then look in the other box. Children were prompted with the 
words "Point to a box for me to look in." This was repeated for six training trials. As a check, after the first box was opened on trial six, the child was asked "So who gets the treat this time?" The training phase concluded when children gave three correct responses to this question (all children passed the training phase).

For the testing phase, children completed 12 trials following the same procedure, except that transparent boxes were used. After each trial, the experimenter announced in a neutral tone "[I/you] keep the treat this time," as appropriate. Children used the same response mode in the training and testing phases.

\section{Counterfactual Reasoning Task}

This task was adapted from Beck et al. (2011). There were four test stories in total. In each story, an event occurred that caused a protagonist to act in a particular way. For example, in one story it starts to rain, so a person in a park goes indoors to avoid the rain. The child was then asked "What if it hadn't rained-where would [they] be?" Stories were accompanied by pictures showing the events described. For each story, children responded by pointing to one of two pictures: one picture showed the current situation (e.g., the character indoors), and one showed an alternative situation (e.g., the character in the park).

\section{False Belief Task}

This task was adapted from Carroll et al. (2012). There were four test stories in total. In each story, a protagonist initially held a true belief; then an event occurred that caused that belief to become false (e.g., an item was moved from one location to another). The child was then asked where the protagonist thought the object was. For each trial, the child was able to choose between two pictures to indicate their response. One picture showed the current situation (e.g., the object in its current location), and one showed an alternative situation (e.g., the object in its original location).

\section{Results}

We hypothesized that there would be significant effects of changing response mode, and of introducing a delay. For ease of comparison across tasks, analyses were conducted on percentage accuracy scores (accuracy by task and condition is shown in Figure 1; note that arcsine transforming the data did

100

80

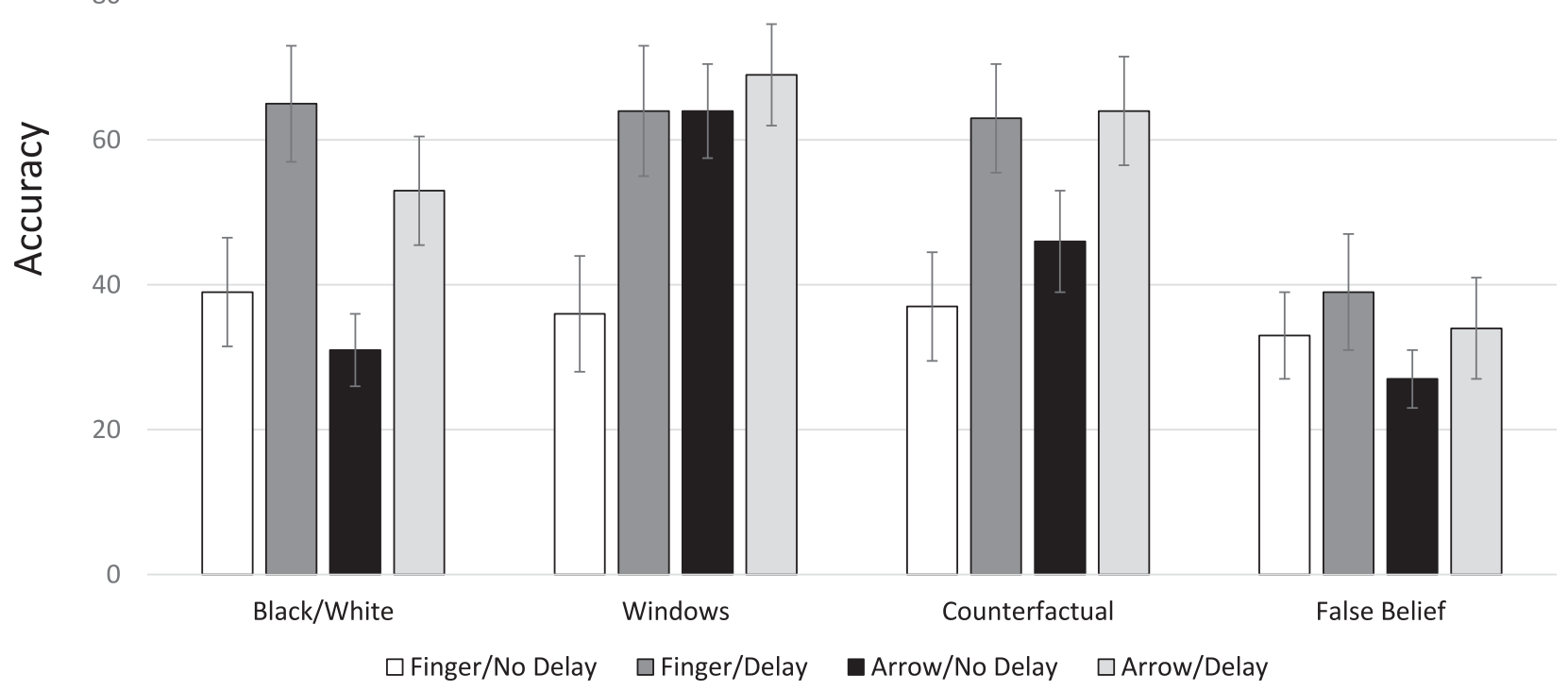

Task

Figure 1. Accuracy (\% correct) on task by condition, with standard error bars. 
Table 1

Response Delay and Response Mode Effects by Task

\begin{tabular}{clc}
\hline & \multicolumn{1}{c}{$\begin{array}{c}\text { Response delay } \\
\text { effect? }\end{array}$} & $\begin{array}{c}\text { Response mode } \\
\text { effect? }\end{array}$ \\
\hline Black-White task & Yes & No \\
& $F(1,77)=8.10$, & $F(1,77)=1.47$, \\
& $p=.006, \eta_{\mathrm{p}}^{2}=.095$ & $p=.230, \eta_{\mathrm{p}}^{2}=.019$ \\
Windows task & $\mathrm{Yes}^{\mathrm{a}}$ & $\mathrm{Yes}^{\mathrm{a}}$ \\
& $F(1,92)=4.91$, & $F(1,92)=4.91$, \\
Counterfactual & $p=.029, \eta_{\mathrm{p}}^{2}=.051$ & $p=.029, \eta_{\mathrm{p}}^{2}=.051$ \\
Reasoning task & $\mathrm{Yes}^{2}$ & No \\
False Belief task & $p<.92)=17.9$, & $F(1,92)=1.170$, \\
& No & $p=.282, \eta_{\mathrm{p}}^{2}=.013$ \\
& $F(1,92)=0.548$, & No \\
& $p=.461, \eta_{\mathrm{p}}^{2}=.006$ & $p(1,92)=0.367$, \\
\end{tabular}

${ }^{a}$ If Bonferroni corrections are made for the four follow-up analyses of variance (alpha: $p=.0125$ ), then the Response Delay and Response Mode effects on the Windows task become nonsignificant. However, we suggest that interpretation would misrepresent children's performance. The Windows task shows the largest overall effect of response manipulation $(p=.001$, $\left.\eta_{\mathrm{p}}^{2}=.136\right)$, and since the Delay and Response Mode effects are of similar size (both $\eta^{2}=.051$ ), we conclude that both should be regarded as significant. We suggest that the lack of significance in the post hoc comparisons is a type II error arising from a conservative Bonferroni correction, and that the appropriate interpretation is that Windows task performance is improved both by delay and alternative response modes.

not change the pattern of results). To investigate the effects of Response Delay and Response Mode, a $2 \times 2 \times 4$ mixed ANOVA was conducted, with Response Delay (Delay, No Delay) and Response Mode (Finger, Arrow) as between-child factors, and Task (Black-White task, Windows task, Counterfactual Reasoning task, False Belief task) as a withinchild factor. There was a significant main effect of Response Delay, $F(1,77)=10.46, p=.002, \eta_{\mathrm{p}}^{2}=.12$. There was no main effect of Response Mode, $F(1$, $77)=0.15, p=.697, \eta_{p}^{2}=.002$, and no significant interaction between Response Delay and Response Mode, $F(1,77)=0.33, p=.565, \eta_{\mathrm{p}}^{2}=.004$. There was a significant main effect of Task, $F(3,231)=73.47$, $p<.001, \eta_{\mathrm{p}}^{2}=.49$. There were also significant interactions between Task and Response Delay, $F(3$, 231) $=3.21, p=.024, \eta_{p}^{2}=.04$, and between Task and Response Mode, $F(3,231)=3.50, p=.016$, $\eta_{\mathrm{p}}^{2}=.04$. There was, however, no significant threeway interaction between Task, Response Delay, and Response Mode, $F(3,231)=0.22, p=.88$.

To follow up these interactions, four two-way between-child analyses of variance were conducted, one for each task, with Response Delay (Delay, No Delay) and Response Mode (Finger, Arrow) as factors (see Table 1). In summary, introducing a delay improved performance on three of the four tasks (the Black-White, Windows, and Counterfactual Reasoning tasks-but not the False Belief task). Using a nonstandard response mode improved performance on only one task (the Windows task). There were no significant interactions between Response Delay and Response Mode. Performance on all tasks improved with age, though there were no interactions between age and either response manipulation (see Tables S3-S6).

\section{Discussion}

This study investigated whether children's performance on tasks involving inhibitory control can be boosted by changing the way children make their responses. It compared the effects of varying Response Delay and Response Mode across four cognitive domains, standardizing both the means of delay and responding, and using a single sample of children. Overall, a significant main effect was observed for Response Delay, but not for Response Mode. Separate task analyses revealed that Response Delay improved performance on three tasks (the Black-White, Windows, and Counterfactual Reasoning tasks-but not the False Belief task). This improvement was independent of whether preschoolers responded using their finger or an arrow. In contrast, a Response Mode boost was seen only in the Windows task. These results clarify our understanding of the way that changes in response influence inhibitory performance: Delaying responding can provide a generalizable benefit, but changing the way children respond boosts performance only in limited circumstances.

\section{Response Delay Effects}

The present findings replicate the Response Delay effect previously observed with the Counterfactual Reasoning task (Beck et al., 2011) and DayNight task (Montgomery \& Fosco, 2012). Importantly, they go well beyond this, providing evidence of a Response Delay effect in the Black-White task and Windows task. It is now clear that genuine benefits from a Response Delay are found on several tasks-two pure measures of inhibitory control (the Black-White and Day-Night tasks) and two different reasoning tasks with inhibitory demands (the Windows and Counterfactual Reasoning tasks). These data suggest that delaying responding can $d i-$ rectly improve preschoolers' performance on a range of tasks requiring inhibitory control. 
It would nevertheless be premature to conclude that delay improves performance on all measures of inhibitory control. In prior research, Go/No-go task performance did not improve following a delay (Barker \& Munakata, 2015). This contrasting result initially appears hard to explain, since the Go/Nogo task relies on inhibitory control-one might therefore expect delays to improve performance. However, the Active Computation account (Diamond et al., 2002) offers a way to reconcile these findings. It proposes that delay improves performance by providing time for children to work out the correct response. In the Black-White, Day-Night, Windows and Counterfactual Reasoning tasks, children need to work out the correct response-and delay improves performance. Conversely, on the Go/No-go task, there is no correct response to work out (the task has a single response-no response is made on inhibitory, No-go trials), and delay does not improve performance. Thus, the Active Computation account can explain why delay improves performance on most inhibitory tasks, but not the Go/No-go task. Conversely, the Passive Dissipation account (Simpson \& Riggs, 2007), which posits that delay allows the activation of the incorrect prepotent response to fade, cannot explain why delay fails to improve performance on the Go/Nogo task. The Go/No-go task does contain an incorrect prepotent response, and so according to the Passive Dissipation account, delay should allow this prepotency to fade, leading to better task performance. The poor performance reported in Barker and Munakata's (2015) study shows clearly that this was not the case.

Uniquely among the present tasks, there was no Response Delay effect in the False Belief task. The task clearly requires the child to work out the correct response, so it may seem surprising that additional time provided by a delay did not help. One possible explanation is that preschoolers fail the False Belief task because they do not yet have an explicit understanding of false beliefs-that is, they lack full conceptual understanding that other people can have beliefs that are false, or different from their own. There is evidence that infants have an implicit understanding of mental states (Baillargeon, Scott, \& He, 2010), although interpretations of this evidence differ (Heyes, 2014). What does seem clear is that implicit knowledge of mental states develops before the explicit understanding required to pass the standard False Belief task. We suggest that without this explicit understanding, it did not matter how long children were given on our False Belief task. They were simply unable to work out the correct response. (By extension, it is plausible that delay could improve False Belief performance in older children, who would like to have a slightly better grasp of mental states than children in the current sample.)

In contrast, counterfactual reasoning performance was improved by a delay, consistent with the idea that these children possess the conceptual knowledge that changes to past events can lead to altered outcomes, and that delays provide the time they need to act on that knowledge. The crucial difference between counterfactual reasoning and false belief reasoning, we suggest, is presence versus absence of explicit knowledge: children do understand that changes to past events can lead to changes in the present, but they don't yet explicitly understand that other people's mental states can differ from their own. If children lack explicit conceptual knowledge, then a manipulation that helps them to act on that knowledge will not improve performance.

\section{Response Mode Effects}

Surprisingly, the Response Mode effect was limited, boosting performance only on a single task. This study replicated the robust Response Mode effect reported with the Windows task. However, it did not replicate the Response Mode effect observed with the Counterfactual Reasoning task (Beck et al., 2011). In this study, children responded using a handheld arrow; conversely, Beck et al.'s study used a rotating arrow. As Beck and colleagues noted, using a rotating arrow may also have introduced a delay. If a Response Mode effect is only seen when the nonstandard response slows responding, but not when responding happens at normal speed, this suggests that the improved performance should be attributed to a Response Delay effect. In other words, the apparent Response Mode effect seen in the Counterfactual Reasoning task is simply a Response Delay effect in disguise.

Thus, the benefits of changing response mode seem to be limited-perhaps restricted only to the Windows task. Notably, this beneficial effect is apparent even with response modes that do not delay responding. What is still unclear is why the Windows task is particularly susceptible to the Response Mode effect. One potential explanation relates to children's expectations about the act of pointing itself. Several studies show that children expect pointing to be veridical, and they have difficulty interpreting pointing when it is used deceptively or incorrectly (Couillard \& Woodward, 1999; 
Palmquist, Burns, \& Jaswal, 2012). Children's poor performance on the standard Windows task may reflect their expectations that pointing inherently serves a veridical purpose (e.g., to point to the desired treat). It may be that minor changes in the pointing response remove these expectations. If so, any deviation from standard veridical pointing should free children from those expectations, allowing them to act more freely.

\section{Conclusion}

The present results clarify how changes in the way children respond can improve their inhibitory control. Introducing a delay boosts performance, with a plausible mechanism being that delays assist children in working out the correct response-in other words, an externally introduced delay can free children from their own tendency to respond too quickly, before they have worked out their response. This raises the possibility that a crucial part of the development of inhibitory control is through children becoming better able to regulate their own speed of responding (Atance, Bernstein, \& Meltzoff, 2010; Simpson et al., 2019). In contrast, the benefits of changing response mode appear more limited. Future interventions seeking to support children in overcoming prepotent responses would have a greater likelihood of success if they focus on delaying responses. The present data suggest that doing so would boost performance on any inhibitory task in which children must select between different responses-though future work should determine whether externally imposed delays can also boost performance in other, noninhibitory domains.

\section{References}

Atance, C. M., Bernstein, D. M., \& Meltzoff, A. N. (2010). Thinking about false belief: It's not just what children say, but how long it takes them to say it. Cognition, 116, 297-301. https://doi.org/10.1016/j.cognition.2010. 05.008

Baillargeon, R., Scott, R. M., \& He, Z. (2010). False-belief understanding in infants. Trends in Cognitive Sciences, 14, 110-118. https://doi.org/10.1016/j.tics.2009.12.006

Barker, J., \& Munakata, Y. (2015). Time isn't of the essence: Activating goals rather than imposing delays improves inhibitory control in children. Psychological Science, 26, 1898-1908. https://doi.org/10.1177/ 0956797615604625

Beck, S. R., Carroll, D. J., Brunsdon, V. E. A., \& Gryg, C. K. (2011). Supporting children's counterfactual thinking with alternative modes of responding. Journal of Experimental Child Psychology, 108, 190-202. https://doi. org/10.1016/j.jecp.2010.07.009

Beck, S. R., Riggs, K. J., \& Gorniak, S. L. (2009). Relating developments in children's counterfactual thinking and executive functions. Thinking and Reasoning, 15, 337354. https://doi.org/10.1080/13546780903135904

Carlson, S. M., \& Moses, L. J. (2001). Individual differences in inhibitory control and children's theory of mind. Child Development, 72, 1032-1052. https://doi. org/10.1111/1467-8624.00333

Carlson, S. M., Moses, L. J., \& Hix, H. (1998). The role of inhibitory processes in young children's difficulties with deception and false belief. Child Development, 69, 672-691. https://doi.org/10.1111/j.1467-8624.1998.tb 06236.x

Carroll, D. J., FitzGibbon, L., \& Critchley, A. (2014). Why do alternative ways of responding improve children's performance on tests of strategic reasoning? British Journal of Developmental Psychology, 32, 195-204. https:// doi.org/10.1111/bjdp.12033

Carroll, D. J., Riggs, K. J., Apperly, I. A., Graham, K., \& Geoghegan, C. (2012). How do alternative ways of responding influence 3- and 4-year-olds' performance on tests of executive function and theory of mind? Journal of Experimental Child Psychology, 112, 312-325. https://doi.org/10.1016/j.jecp.2012.03.001

Couillard, N., \& Woodward, A. (1999). Children's comprehension of deceptive points. British Journal of Developmental Psychology, 17, 515-521. https://doi.org/10. 1348/026151099165447

Diamond, A., Kirkham, N., \& Amso, D. (2002). Conditions under which young children can hold two rules in mind and inhibit a prepotent response. Developmental Psychology, 38, 352-362. https://doi.org/10.1037/00121649.38.3.352

Eckert, H. M., \& Eichorn, D. H. (1977). Developmental variability in reaction time. Child Development, 48, 452458. https://doi.org/10.2307/1128638

Faul, F., Erdfelder, E., Lang, A.-G., \& Buchner, A. (2007). G*Power 3: A flexible statistical power analysis program for the social, behavioral, and biomedical sciences. Behavior Research Methods, 39, 175-191. https://doi.org/10.3758/BF03193146

Gerstadt, C. L., Hong, Y. J., \& Diamond, A. (1994). The relationship between cognition and action: Performance of children 3.5-7 years old on a Stroop-like day-night test. Cognition, 53, 129-153. https://doi.org/10.1016/ 0010-0277(94)90068-X

Gilmore, C., Attridge, N., Clayton, S., Cragg, L., Johnson, S., Marlow, N., ... Inglis, M. (2013). Individual differences in inhibitory control, not non-verbal number acuity, correlate with mathematics achievement. PLoS One, 8, e67374. https://doi.org/10.1371/journal.pone.0067374

Hala, S. M., \& Russell, J. (2001). Executive control within strategic deception: A window on early cognitive development? Journal of Experimental Child Psychology, 80, 112-141. https://doi.org/10.1006/jecp.2000.2627 
Heyes, C. (2014). False belief in infancy: A fresh look. Developmental Science, 17, 647-659. https://doi.org/10. 1111/desc. 12148

Kusche, C. A., \& Greenberg, M. T. (1994). The PATHS curriculum. Seattle, WA: Developmental Research and Programs.

Lee, W. S. C., \& Atance, C. M. (2016). The effect of psychological distance on children's reasoning about future preferences. PLoS One, 11, e0164382. https://doi.org/ 10.1371/journal.pone.0164382

Ling, D., Wong, C. D., \& Diamond, A. (2016). Do children need reminders on the Day-Night task, or simply some way to prevent them from responding too quickly? Cognitive Development, 37, 67-72. https://doi.org/10. 1016/j.cogdev.2015.10.003

Montgomery, D. E., \& Fosco, W. (2012). The effect of delayed responding on Stroop-like task performance among preschoolers. The Journal of Genetic Psychology, 173, 142-157. https://doi.org/10.1080/00221325.2011.583699

Murray, K. T., \& Kochanska, G. (2002). Effortful control: Factor structure and relation to externalizing and internalizing behaviors. Journal of Abnormal Child Psychology, 30, 503-514. https://doi.org/10.1023/A:1019821031523

Palmquist, C. M., Burns, H. E., \& Jaswal, V. K. (2012). Pointing disrupts preschoolers' ability to discriminate between knowledgeable and ignorant informants. Cognitive Development, 27, 54-63. https://doi.org/10.1016/ j.cogdev.2011.07.002

Petersen, I. T., Hoyniak, C. P., McQuillan, M. E., Bates, J. E., \& Staples, A. D. (2016). Measuring the development of inhibitory control: The challenge of heterotypic continuity. Developmental Review, 40, 25-71. https:/ / doi.org/ 10.1016/j.dr.2016.02.001

Russell, J., Hala, S., \& Hill, E. L. (2003). Mechanising an executive task: The performance of preschool children, children with autism and with moderate learning difficulties in the automated Windows Task. Cognitive Development, 18, 111-137. https://doi.org/10.1016/ S0885-2014(02)00163-6

Sabbagh, M. A., Moses, L. J., \& Shiverick, S. (2006). Executive functioning and preschoolers' understanding of false beliefs, false photographs, and false signs. Child Development, 77, 1034-1049. https://doi.org/10.1111/j. 1467-8624.2006.00917.x

Simpson, A., Al Ruwaili, R., Jolley, R., Leonard, H., Geeraert, N., \& Riggs, K. J. (2019). Fine motor control underlies the association between response inhibition and drawing skill in early development. Child Development, 90, 911-923. https://doi.org/10.1111/cdev. 12949

Simpson, A., \& Riggs, K. J. (2007). Under what conditions do young children have difficulty inhibiting manual actions? Developmental Psychology, 43, 417-428. https:/ / doi.org/10.1037/0012-1649.43.2.417

Simpson, A., \& Riggs, K. J. (2009). What makes responses prepotent for young children? Insights from the Grass/ Snow task. Infant and Child Development, 18, 21-35. https:/ / doi.org/10.1002/icd.576

Wimmer, H., \& Perner, J. (1983). Beliefs about beliefs: Representation and constraining function of wrong beliefs in young children's understanding of deception. Cognition, 13, 103-128. https://doi.org/10.1016/00100277(83)90004-5

\section{Supporting Information}

Additional supporting information may be found in the online version of this article at the publisher's website:

Table S1. A Taxonomy of Incidental Demands for the Four Tasks

Table S2. Counterbalancing Orders for Task Administration

Table S3. Younger Group's Mean Correct Responses on Each Task in Delay and No Delay Conditions (Standard Deviations in Brackets)

Table S4. Older Group's Mean Correct Responses on Each Task in Delay and No Delay Conditions (Standard Deviations in Brackets)

Table S5. Younger Group's Mean Correct Responses on Each Task in Finger and Arrow Conditions (Standard Deviations in Brackets)

Table S6. Younger Group's Mean Correct Responses on Each Task in Finger and Arrow Conditions (Standard Deviations in Brackets)

Appendix S1. Instructions for Response Modes

Appendix S2. Task Scripts

Appendix S3. Descriptive Data 